\title{
Liquid migration in flowing granular materials
}

\author{
Thijmen Hagen ${ }^{1,2, *}$, Stefan Luding ${ }^{1}$, Devaraj van der Meer ${ }^{2}$, Vanessa Magnanimo ${ }^{3}$, and Ahmed Jarray ${ }^{1, * *}$ \\ ${ }^{1}$ Multi-Scale Mechanics (MSM), TFE, ET, MESA+, Institute for Nanotechnology, University of Twente, The Netherlands. \\ ${ }^{2}$ Physics of Fluids Group, MESA+, TNW, J.M. Burgers Center for Fluid Dynamics, University of Twente, The Netherlands. \\ ${ }^{3}$ Construction Management and Engineering (CME), Faculty of Engineering Technology, University of Twente, The Netherlands.
}

\begin{abstract}
In partially wet granular beds, liquid migrates between particles due to collisions and contacts. This, in turn, influences the flow behaviour of the granular bed. We investigate liquid redistribution in moving monodisperse particles in a rotating drum using Discrete Element Method (DEM) simulations. For weak capillary forces, liquid re-distribution, induced by the continuous flow of particles, leads to concentration of the liquid in the core of the bed, where the flow is quasi-static. High capillary forces reduce the surface flow speed and granular temperature. This decreases liquid bridges rupturing in the flowing layer, allowing the liquid to remain in the outer region of the bed.
\end{abstract}

\section{Introduction}

Unsaturated wet granular assemblies are composed of a network of inter-particles mechanical contacts and liquid bridges connecting adjacent particles. These bridges form and rupture in the pendular regime $[1,2]$, giving rise to capillary forces and liquid migration upon opening contacts between particles, which in turn affects the flow behaviour of the granular system [1]. Conversely, the flow behaviour and the shearing occurring within the granular system determine the liquid distribution and transport between particles [3]. This complex phenomenon happens both in nature and many industrial processes associated with particulate systems, and thus has an enormous technological and environmental importance. For instance, an excessive increase of the moisture in soil weakens its strength and may cause landslides or avalanches [4]. In industry, liquid migration can be found in pharmaceutical applications such as spray coating of tablets and granulation processes, where an in-homogeneous liquid distribution can deteriorate the quality of the final product [5]. Yet, the few available liquid migration models are built on various approximations, and their parameterization is based on empirical testing or assumptions, mainly because their experimental observation is extremely challenging $[6,7]$. Furthermore, it is still unclear how to deal with liquid redistribution upon bridge rupture, and how this interplays with the flow behaviour of granular media.

In this work, using DEM simulations, we examined the effect of capillary forces and liquid transfer on liquid distribution and flow behaviour of particles in a rotating drum apparatus. For this, adequate parameter values of the recently modified version of Mani's liquid bridge model $[6,8]$ were identified. Liquid migration in the moving par-

\footnotetext{
*e-mail: thijmenhagen@gmail.com

**e-mail: a.jarray@utwente.nl

A video is available at https://doi.org/10.48448/r3th-bb77
}

ticles bed was simulated and explained in terms of the flow dynamics and liquid bridges rupture.

\section{Methods}

\subsection{DEM simulations}

Discrete Elements Method (DEM) simulations were performed using MercuryDPM code [9], with the same drum, particles, and liquid properties as in the experiments performed by Jarray et al. [1, 10]. The drum was filled to 35\% of its volume with monodisperse glass particles, of density $\rho=2500 \mathrm{~kg} / \mathrm{m}^{3}$ and radius $r=1.25 \mathrm{~mm}$. Properties of the drum and particles are shown in Table 1. DEM simulation were performed for a duration of $10 \mathrm{~s}$, with a time step $\Delta t=2.5 \cdot 10^{-6} \mathrm{~s}$, ensuring a stable system. Similarly to the hydrophobic coating applied on the drum walls in the experiments $[1,10]$, liquid bridges formation between particles and the walls of the drum were switched off.

Table 1. Properties of the drum and the particles.

\begin{tabular}{ll}
\hline Parameter & Value \\
\hline Radius of the drum, $R[\mathrm{~m}]$ & 0.0605 \\
Width of the drum, $w[\mathrm{~m}]$ & 0.022 \\
Radius of the particles, $r[\mathrm{~mm}]$ & 1.25 \\
Density, $\rho\left[\mathrm{kg} / \mathrm{m}^{3}\right]$ & 2500 \\
Young' $s$ modulus of the particles, $E[\mathrm{MPa}]$ & 63 \\
Poisson's ratio of the particles, $v[-]$ & 0.21 \\
\hline
\end{tabular}

\subsection{Liquid migration model}

The liquid is initially added as liquid films on the particles, following an updated version of Mani's liquid migration model [8], recently proposed by Roy et al. [6]. When two particles come into contact, a capillary bridge forms between them. The amount of liquid, initially present on 


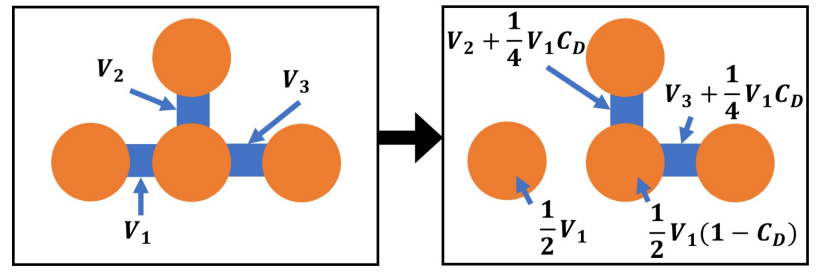

Figure 1. Schematic explanation of the liquid bridge rupture and liquid migration between particles. Adapted from Refs. [6, 8].

the film, is then poured to the new capillary bridge. Roy et al. [6] proposed to use a maximum capillary bridge volume given by

$$
V_{\max }=\beta r^{3},
$$

where $r$ is the particle radius in the monodisperse particles bed, and $\beta$ is the maximum bridge volume coefficient. If the total available liquid, $V_{e}$, is less than $V_{\max }$, all liquid is moved to the capillary bridge. If $V_{e}>V_{\max }$, the liquid is moved from each particle in proportion to the liquid film volume, and the excess liquid remains in the liquid film [6]. The capillary bridge exerts an adhesive force on the particles it connects given by

$$
f_{i j}^{c}=\frac{f_{\max }^{c}}{1+1.05 \bar{S}+2.5 \bar{S}^{2}},
$$

where $\bar{S}=S \sqrt{r / V_{b}}$ is the separation distance $S$ normalized by the square root of the ratio of the particle radius and the bridge volume $V_{b}$. The maximum capillary force, which occurs when the particles are in contact and in the presence of a liquid bridge, $S=0$, is independent of $V_{b}$ and given by

$$
f_{\text {max }}^{c}=2 \pi r \sigma \cos \theta,
$$

where $\sigma$ is the surface tension of the liquid and $\theta$ the liquid-particle contact angle. When the two particles connected by a capillary bridge move farther apart than the critical separation distance

$$
S_{c}=\left(1+\frac{\theta}{2}\right) V_{b}^{1 / 3},
$$

the bridge ruptures, and the capillary bridge liquid is distributed between the two particles. A fraction $C_{D}$ of the liquid volume is distributed evenly between the existing bridges. The remaining fraction, $1-C_{D}$, is stored in the particle's liquid film as shown schematically in Fig. 1. $C_{D}=1$ means that most particles will have no available liquid in their liquid film, and existing bridges will grow much faster than for lower values of $C_{D}$.

\section{Results and discussion}

\subsection{Dry case}

DEM simulations were performed with the same conditions and material properties as in the experiments by Jarray et al. [1]. Fig. 2 compares the experimental and numerical mean angle of repose as a function of the rotation speed $\Omega$ and the Froude number defined as

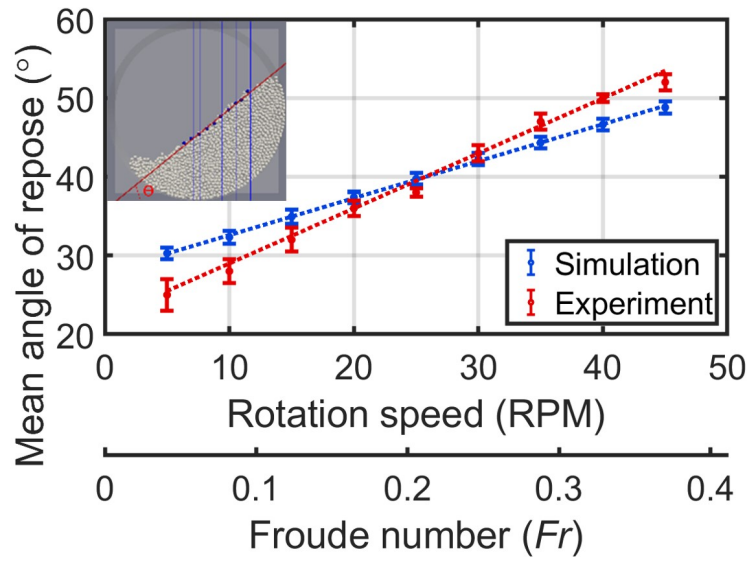

Figure 2. Experimental vs. DEM simulation of the angle of repose as a function of rotation speed and Froude number $(\mathrm{Fr})$ for the dry case. Experimental data were obtained from Ref. [1].

$$
F r=\sqrt{\frac{\Omega^{2} R}{g}},
$$

which describes the ratio of centrifugal to gravitational acceleration. The mean angle of repose is calculated using an in-house python code that takes the average of the angle of repose over the last 6 seconds of simulations, where the continuous flow is reached. A small discrepancy is observed, but the same increasing trend is obtained in both experiments and simulations. For low rotation speeds, at 5 RPM, the measured angle in the simulations is about $5^{\circ}$ higher than experiments, while for high rotation speeds, at $45 \mathrm{RPM}$, it is lower by $5^{\circ}$. Since the best agreement was obtained for 25 RPM, we will use this rotation speed for the simulation of the wet case in the following Section 3.2.

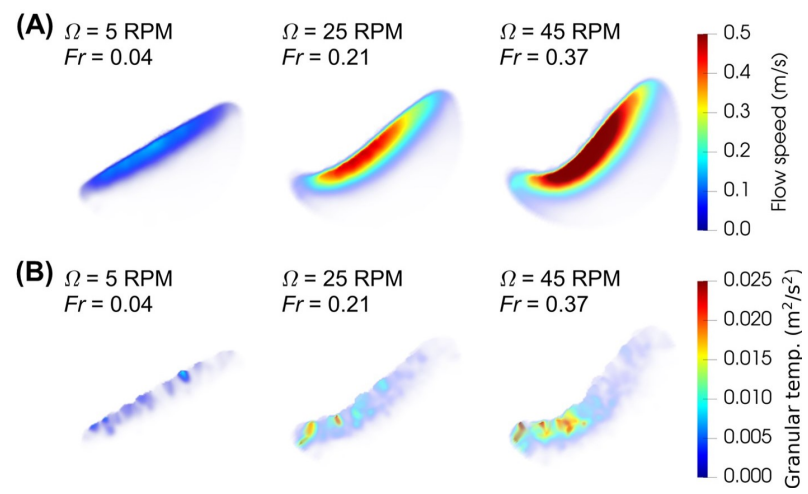

Figure 3. A) flow speed, and B) granular temperature averaged over the last 0.3 seconds of the DEM simulations of the dry case at various rotation speeds. The white zone below the flowing layer indicates particles at rest in the rotating reference frame.

Fig. 3 (A) shows the flow speed $\left|\vec{\phi}_{i}\right|$ of the particulate bed at various rotation speeds. The flow speed is the magnitude of the velocity of the particle relative to the drum, and was averaged over the transverse direction of the drum, and over the last 0.3 seconds of the simulation. $\left|\overrightarrow{\phi_{i}}\right|$ of particle $i$ is calculated as

$$
\left|\overrightarrow{\phi_{i}}\right|=\left|\overrightarrow{v_{i}}-\overrightarrow{v_{\Omega, i}}\right|
$$


where $\overrightarrow{v_{i}}$ is the velocity of particle $i$ and $\overrightarrow{v_{\Omega, i}}$ is its angular velocity induced by the rotation of the drum. When the rotation speed is low (5 RPM), the bed surface is flat and the flowing layer is narrow with low velocity. For higher rotation speeds (25 and 45 RPM), the particles move upwards with more inertial energy induced by the rotating drum, resulting in an S-shaped surface of the bed. The flowing layer becomes wider and the particles have higher velocities. From the flow speed, the granular temperature $T_{i}$ of particle $i$ can be calculated as

$$
T_{i}=\frac{1}{2}\left|\overrightarrow{\phi_{i}}-\overrightarrow{\phi_{a v}}\right|^{2}
$$

where $\overrightarrow{\phi_{a v}}$ is the local average of the flow speed of the particles within 3 particle radii from a reference particle $i$ [11]. The granular temperature is a measure of the relative motion between particles. $T_{i}$ is small for regions where the particles behave like a solid, while it is high for regions where the particles flow and collide. From Fig. 3 (B), an increase in the rotation speed leads to an increase in the granular temperature, especially in bottom-left region of the flowing layer where the avalanche impacts on the base of the slope, indicating higher number of collisions.

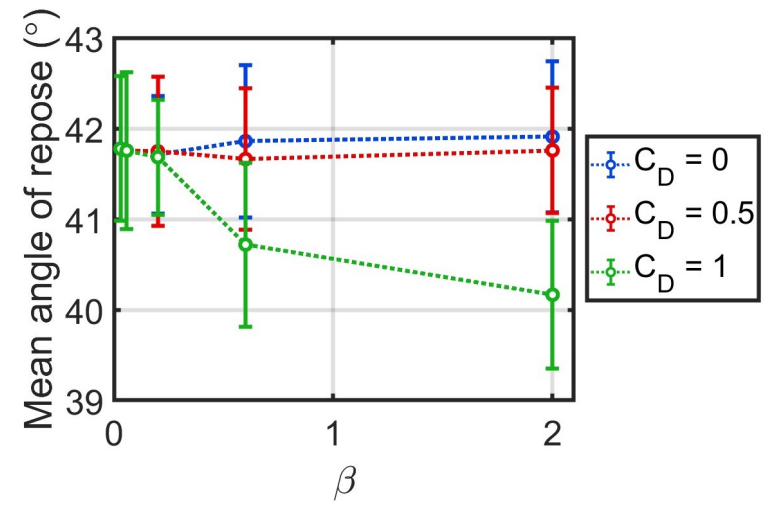

Figure 4. Angle of repose obtained from DEM simulations as a function of $\beta$ for different values of $C_{D}$.

\subsection{Wet case}

A small amount of liquid inducing an average capillary bridge force of $F_{c}=0.1372 \mathrm{mN}$ between particles (the same as case 4 in the experiments performed by Jarray et al. [1]) was introduced in the simulations. Two liquid migration parameters need to be determined; the maximum bridge volume $\beta$ (see eq. 8) and the coefficient of distribution $C_{D}$, which quantifies the distribution of liquid after a bridge rupture. Fig. 4 shows the mean angle of repose plotted against $\beta$ for $C_{D}=0,0.5$ and 1 , which is is not significantly affected by $\beta$, except for $C_{D}=1$, where a slight decrease is observed with increasing $\beta$.

We explore next the effect of $C_{D}$ and $\beta$ on the distribution of liquid bridges within the bed. In Figures 5 (A) and (B), we plot the probability distribution function (PDF) of liquid bridge volume for various $\beta$, and for $C_{D}=0.5$ and 1 , respectively. For the smallest two values of $\beta, 0.03$ and 0.058 , the PDF shows a single peak. This means that the volumes of all the non-empty bridges is equal to the maximum bridge volume. The PDF becomes smoother
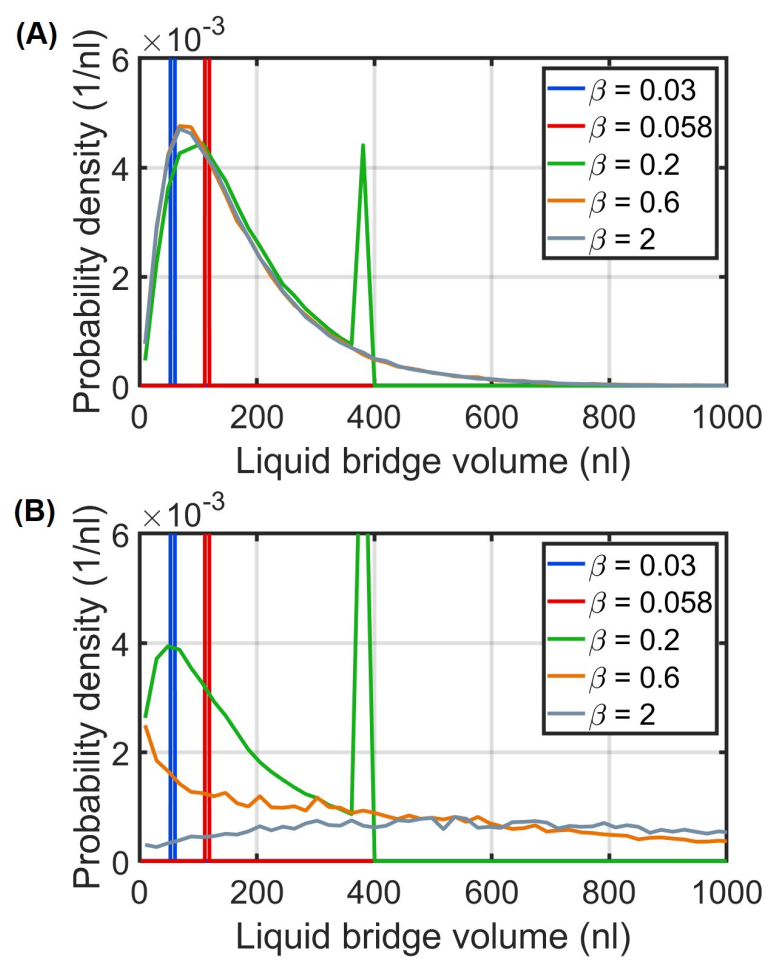

Figure 5. Probability density function of the capillary bridge volumes for various values of maximum bridge volume $\beta$, and for liquid distribution coefficient (A) $C_{D}=0.5$, and (B) $C_{D}=1$.

and positively skewed for higher $\beta$ values, indicating that a bridge is formed at each particle-particle contact point, i.e., all possible bridges are formed. This happens for $\beta=0.6$ with both $C_{D}=0.5$ and 1 . Higher $\beta$ values give almost the same distribution, but increase the computation time. Thus, $\beta=0.6$ is the optimal value and will be used throughout the rest of this paper. For $\beta \geq 0.6$ and $C_{D}=1$ in Fig. 5 (B), the number of bridges is about 4 times smaller than for $C_{D}=0.5$ (in Fig.5 (A)), and the probability density function is spread out to much higher volumes, indicating that most particles have high amount of liquids in their capillary bridge. Since there should be some liquid in each particle (i.e., not all liquid should be transferred to the bridges), $C_{D}=1$ is not a realistic value, and it is more reasonable to use $C_{D}=0.5$.

Now that the reasonable values of $C_{D}$ and $\beta$ are identified, we can study the mean angle of repose as a function of the capillary force and the granular Bond number $(\mathrm{Bo})$. $B o$ is defined as the ratio of the capillary force to the gravitational force of a single particle,

$$
B o=\frac{3 \sigma \cos \theta}{2 r^{2} \rho g} \text {. }
$$

In Fig. 6, the mean angle of repose is plotted against the capillary force and $B o$. At low Bo, agreement was observed between experiments and simulations. However, for large $B o$, the mean angle of repose in the experiments is higher than in the simulations, with a maximum deviation of $6^{\circ}$. This could be attributed to inconsistencies in the experimental setup, or shortcomings in the liquid migration model requiring additional mechanisms and parameters. 


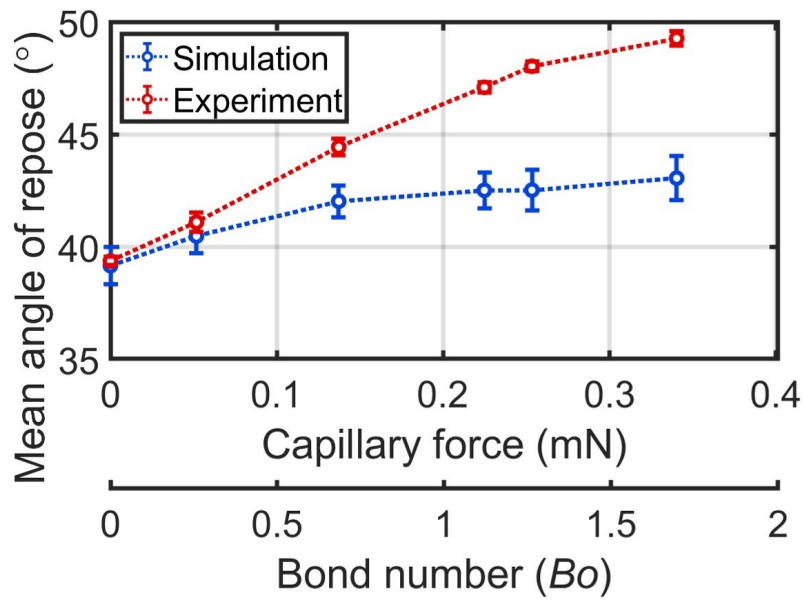

Figure 6. Experimental vs. numerical angle of repose as a function of the capillary force and the Bond number. Experiments are obtained from Ref. [1].

Figures 7 (A), (B), (C) and (D) show, respectively, the flow speed, granular temperature, strain rate, and liquid distribution profiles, averaged over the last 0.3 seconds of the DEM simulations for different Bond numbers. For low $B o$, there is a thin fast flow layer on top of the bed. When increasing the capillary forces, this flowing layer gets slower and thicker, because particles stick together. An increase of the capillary forces, reduces the granular temperature (see Fig. 7 (B)) as particles experience less collisions and strain rate. The same observations were obtained experimentally by Jarray et al. [1]. Strain rate is the highest at the base of avalanches (i.e., left side of the bed in Fig. $7(\mathrm{C})$ ). An increase of $B o$ reduces the strain rate and makes its distribution more uniform in the flowing layer, with its maximum moving deeper below the surface. Dark blue zones are indicative of big particle clumps moving down as the drum rotates. From Fig. 7 (D), for low Bo, we observe more lquid in the center and depletion outside. This is because liquid bridges are less likely to rupture in this region. This also can be observed in the base of the flowing layer, where the light transparent blue color indicates lower amount of liquid but higher number of particles collisions. However, at higher capillary forces, there are less rupturing of liquid bridges on the flowing layer. As a consequence, liquid tend to migrate more to the outer region of the bed with increasing $B o$.

\section{Conclusion}

We studied the effect of liquid migration parameters on the liquid distribution within a moving granular bed in a rotating drum. Without calibration, the simulations match the experiments of Jarray et al. [1] for intermediate rotation speeds in the dry case. Concerning the effect of capillary forces on the flow behavior of particles in the bed; simulations showed that capillary forces thicken the flowing layer, and reduce the flow speed, particle-particle collisions and thus, liquid bridges rupturing. We found that for low capillary forces, liquid migrates farther from higher strain rate zones towards the core of the granular bed where the flow is quasi-static and only few bridges rupture. Conversely, for high capillary forces, the liquid is more concentrated in the outer regions of the bed, since bridges rupture closer to the core - away from the free surface. Quantitative calibration of the liquid migration model could further improve the DEM predictions.

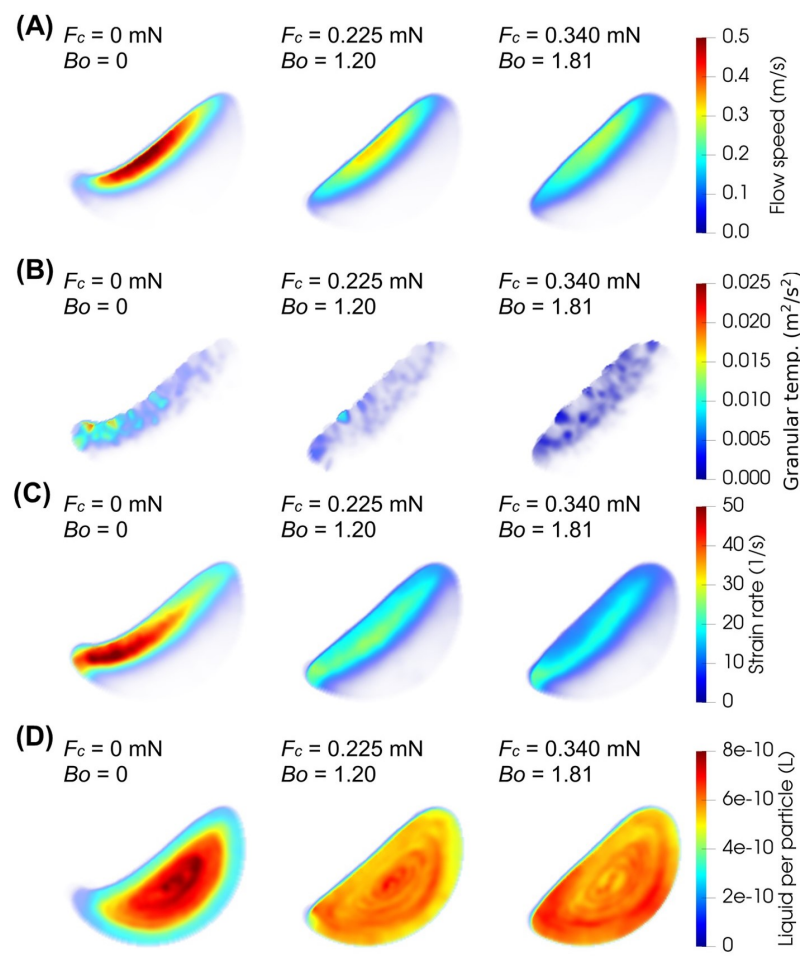

Figure 7. (A) flow speed, (B) granular temperature, (C) strain rate, and (D) liquid distribution, obtained using DEM simulations for various capillary forces and for $F r=0.21$.

We thank H. Shi, T. Weinhart, and S. Roy for their help.

\section{References}

[1] A. Jarray, V. Magnanimo, S. Luding, Powder Technol. 341, 126-139(2019)

[2] M.E.D. Urso, C.J. Lawrence, M.J. Adams, J. Colloid Interface Sci. 220, 42-56 (1999)

[3] R. Mani, D. Kadau, D. Or, H.J. Herrmann, Phys. Rev. Lett. 109, 248001 (2012)

[4] T.W. Van Asch, J. Buma, L.P.H. Van Beek, Geomorphology 30, 25-32 (1999)

[5] R. Turton, Powder Technol. 181, 186-194 (2008)

[6] S. Roy, S. Luding, T. Weinhart, Phys. Rev. E 98, 052906 (2018)

[7] B. Mohan, K. Kloss, J. Khinast, S. Radl, Powder Technol. 264, 377-395 (2014)

[8] R. Mani, D. Kadau, H.J. Herrmann, Granul. Matter 15, 447-454 (2013)

[9] T. Weinhart, T.R. Deepak, M.P. Lantman, C.R. Windows-Yule, et al. Particles V, 123-134 (2017)

[10] A. Jarray, V. Magnanimo, S. Luding, EPJ Web Conf. 140, 03078 (2017)

[11] T. Weinhart, C. Labra, S. Luding, J.Y. Ooi, Powder Technol. 293, 138-148 (2016) 